\title{
Teste de sentar-levantar: apresentação de um procedimento para avaliação em Medicina do Exercício e do Esporte
}

\author{
Claudio Gil Soares de Araújo ${ }^{1}$
}

\section{RESUMO}

A prática regular de atividade física é associada a maior quantidade e qualidade de vida da população. É sabido ainda que a sensação de bem-estar pessoal relaciona-se com a qualidade de vida orientada para a saúde e com a autonomia para a vida. Há consenso de que uma boa aptidão física depende não somente de níveis de potência máxima aeróbica satisfatórios, mas também de padrões apropriados de força e potência muscular, de flexibilidade e de estabilidade postural. É, portanto, conveniente que, do ponto de vista clínico e de saúde pública, existam instrumentos de rastreamento simples e com alta sensibilidade que permitam a avaliação dessas variáveis no âmbito de um consultório. O propósito deste artigo é apresentar o Teste de sentar-levantar (TSL). O TSL consiste, simplesmente, em quantificar quantos apoios (mãos e/ou joelhos ou, ainda, mãos ou antebraços em joelhos), o indivíduo utiliza para sentar e levantar do chão. Atribuem-se notas independentes para cada um dos dois atos - sentar e levantar. A nota máxima é 5 para cada um dos dois atos, perdendo-se um ponto para cada apoio ou ainda meio ponto para qualquer desequilíbrio perceptível. O TSL permite, em pouco tempo e em praticamente qualquer lugar, avaliar vários itens - flexibilidade das articulações dos membros inferiores, equilíbrio, coordenação motora e relação entre potência muscular e peso corporal - de uma vez só, no que talvez possa ser caracterizado como aptidão muscular funcional mínima. Pode ser aplicado em consultórios, escolas, academias e organizações militares. Com base nos resultados do TSL, profissionais de saúde terão provavelmente melhores meios para estimular a adoção de estilos de vida mais ativos e para orientar, de forma mais científica, programas de atividade física.

Palavras-chave: Qualidade de vida relacionada com a saúde. Aptidão física. Testes de aptidão. Potência muscular. Flexibilidade. Autonomia.

\footnotetext{
1. Professor do Programa de Pós-Graduação em Educação Física da UGF; Professor Adjunto do Departamento de Fisiologia da UFF; Médico do Serviço de Medicina do Exercício e do Esporte do HUCFF-UFRJ; Diretor Médico da Clínica de Medicina do Exercício [Clinimex] - Rio de Janeiro, RJ.
}

Endereço para correspondência:

E-mail: cgaraujo@iis.com.br

\section{ABSTRACT \\ Sitting-rising test: introduction of a new procedure for eval- uation in exercise and sports medicine}

Regular physical activity is linked with longer life expectancy and better quality of life. It is already known that wellness is related to health-related quality of life and personal autonomy. There is a consensus that a good physical fitness depends not only on adequate levels of maximal aerobic power, but also on appropriate levels of muscle power and strength, flexibility, and postural stability. Thus, it is convenient that, in the public health and clinical perspectives, there should be simple and highly sensitive screening tools that makes evaluation of these variables in office possible. The purpose of this article is to introduce the Sitting-rising test (SRT). The SRT basically consists in the quantification of the number of support (hands and/or knees, or hands or forearms on knees) one utilizes in order to sit and to rise from the floor. Independent grades are provided to each of the two actions - sitting and rising. The maximal grade is 5 for each one of the actions, losing one point for each support and additional half point for any detectable unbalance. The SRT allows, in very short time and practically in any place, the evaluation of many items - flexibility of lower limb joints, balance, motor coordination, and muscle power/body weight relationship - at the same time, which could be perhaps characterized as minimum functional muscular fitness. Based on the SRT results, health professionals are likely to have better means of stimulating the adoption of more active lifestyles and to advising physical activity programs in a more scientific way.

Key words: Health-related quality of life. Physical fitness. Fitness testing. Muscle power. Flexibility. Autonomy.

Indivíduos fisicamente ativos ou com melhores níveis de aptidão física aeróbica têm menor mortalidade ${ }^{1,2}$. Baseados nesses e em outros dados epidemiológicos consistentes, diretrizes de várias organizações internacionais ${ }^{3-5}$ têm preconizado que toda a população deveria acumular pelo menos $30 \mathrm{mi}-$ nutos de exercício físico na maioria dos dias.

É sabido que a sensação de bem-estar pessoal relaciona-se com a qualidade de vida orientada para a saúde e com a autonomia para a vida ${ }^{6-9}$. Enquanto existem inúmeros questioná- 
rios para determinar ou avaliar a qualidade de vida relacionada com a saúde $7,8,10,11$, os quais incluem questões referentes à prática de atividade física ou à capacidade de executar determinadas ações físicas, parece haver certa dificuldade em associar essas respostas com o que tem sido denominado de variáveis da aptidão física relacionadas com a saúde ${ }^{12}$. Há consenso atualmente de que uma boa condição física depende não somente dos níveis de potência máxima aeróbica satisfatórios, mas também dos padrões apropriados de potência muscular, de flexibilidade e de estabilidade postural ${ }^{5}$. Estas variáveis em níveis apropriados parecem ser importantes para o bem-estar e para a autonomia do indivíduo.

Nas atividades profissionais da área de saúde existe frequientemente a necessidade de atender a um grande número de indivíduos em um período escasso de tempo. Em geral, nessas situações, priorizam-se testes de rastreamento ${ }^{13}$, através dos quais se busca a identificação precoce dos indivíduos que podem ter alguma anormalidade e/ou enfermidade. Desse modo, aqueles que conseguem alcançar os níveis mínimos de corte preconizados são, com grande probabilidade, indivíduos normais ou saudáveis em relação às variáveis medidas ${ }^{14}$. Processos avaliativos de alta sensibilidade, portanto capazes de diferenciar claramente indivíduos com capacidades e/ou estados clínicos distintos, são muito apropriados para situações em que haja grande demanda de atendimento, deixando-se para uma etapa posterior a confirmação eventual e a análise da causa do insucesso na avaliação.

Todo processo de avaliação implica o uso de um método válido e fidedigno, entendendo-se validade como a capacidade de medir aquilo que efetivamente se deseja e fidedignidade, como a qualidade de obter resultados consistentes em repetidas medidas, sejam elas feitas por um único avaliador, em um único dia ou não, ou quando feitas simultaneamente por mais de um avaliador. Em adendo, as principais características de um instrumento de rastreamento para aplicação na área de Medicina do Exercício e do Esporte, em ampla escala, são: rapidez, segurança alta e risco mínimo para o avaliador e o avaliado, simplicidade e não-invasividade, custo unitário baixo ou desprezível, possibilidade de ser realizado em pequeno espaço (por exemplo, a área típica de um consultório), aplicabilidade em todas as populações e faixas etárias e, principalmente, alta sensibilidade, se possível aliada a alta especificidade.

Enquanto existem muitos procedimentos válidos, simples e bem padronizados para a avaliação do componente aeróbico, como, por exemplo, o teste de Cooper ${ }^{15}$, e para a análise da flexibilidade global e por movimentos, tal como o Flexiteste $^{16}$, o mesmo não se pode comentar em relação às variáveis do desempenho muscular funcional. Seria portanto conveniente que, do ponto de vista clínico e de saúde pública, houvesse um instrumento simples e com alta sensibilidade que permitisse a avaliação dessas variáveis no âmbito de um con- sultório convencional, se possível, estabelecendo alguma relação com o peso corporal e a flexibilidade em um contexto funcional específico. Com esse propósito é que estamos descrevendo a seguir o Teste de sentar-levantar (TSL).

\section{DESCRIÇÃO DO TESTE}

\section{Técnica de execução}

O TSL pode ser realizado em praticamente qualquer local, demandando apenas $3 \mathrm{ou} 4 \mathrm{~m}^{2}$ livres e um solo plano e não escorregadio. Para a execução do teste, o indivíduo deverá estar descalço e sem meias e desprovido de vestimentas que possam restringir-lhe a mobilidade. A instrução dada pelo avaliador ao avaliado é muito simples e objetiva: "Procure sentar e levantar, sem se desequilibrar, e utilizando o menor número de apoios que você consiga". Na execução do procedimento, o indivíduo fica em pé, de costas para um colchonete fino ou tapete colocado no solo imediatamente atrás dele, e tenta sentar-se lentamente, sem o apoio das mãos e sem desequilibrarse. O avaliador fica próximo ao avaliado para oferecer-lhe segurança em caso de desequilíbrio, procurando posicionar-se em diagonal, de modo a ter melhor ângulo de visão para identificar o uso de apoios e a presença ou ausência de qualquer desequilíbrio. Completado o ato de sentar é atribuída a nota e solicitado ao avaliado que se levante e novamente o avaliador confere uma nota. Enquanto é válido cruzar as pernas para sentar ou levantar, não se permite que o indivíduo se jogue para trás ao tentar sentar-se. A velocidade de execução não é especificamente medida, mas recomenda-se que o indivíduo realize os dois atos de forma e com velocidade naturais, já que a rapidez relativa não está sendo mensurada e não é observada na avaliação. São normalmente feitas duas tentativas, procurando melhorar o resultado da primeira para a segunda. Caso o avaliador perceba que o indivíduo, por qualquer razão, pode ainda melhorar a técnica de execução, outras tentativas podem ser feitas. Cabe ao avaliador orientar como, eventualmente, o indivíduo poderá melhorar seus resultados, sem pôr em risco sua integridade física. Normalmente, o procedimento é realizado em menos de dois minutos.

\section{Medida}

A medida do TSL consiste simplesmente em quantificar quantos apoios (mãos e/ou joelhos ou, ainda, as mãos sobre os joelhos ou pernas) o indivíduo utiliza para sentar e levantar do chão. Atribuem-se notas independentes para cada um dos dois atos - sentar e levantar. A nota máxima é 5 para cada um dos dois atos. Perde-se ainda meio ponto para qualquer desequilíbrio perceptível. Exemplificando, se o indivíduo para sentar-se sem cair precisa colocar uma das mãos, o resultado é 4 [perda de 1 ponto] e, se para levantar ele tem de utilizar a mesma mão no solo e depois ainda apóia a outra no joelho, temos um resultado de 3 [perda de 2 pontos, um para cada apoio] e assim por diante. Se o indivíduo não consegue sentar 
ou levantar do chão sem auxílio de outra pessoa ou de uma parede, ou ainda necessita de mais de quatro apoios, isto é, precisar ajoelhar, apoiar as duas mãos no solo e ainda apoiar a mão sobre um dos joelhos para levantar, a nota é mínima, isto é, zero. O melhor resultado das duas tentativas para cada um dos atos é escolhido como representativo do indivíduo. Exemplo: nota 4 para sentar e nota 3 para levantar.

\section{Avaliação}

O TSL, um procedimento extremamente simples, permite, em pouco tempo e em praticamente qualquer lugar, avaliar vários itens - flexibilidade das articulações dos membros inferiores, equilíbrio, coordenação motora e relação entre potência muscular e peso corporal - de uma vez só, no que talvez possa ser caracterizado como aptidão muscular mínima. Pode ser aplicado em consultórios, escolas, academias, organizações militares, etc. Tem-se mostrado extremamente fidedigno $^{17-19}$, sensível a variações de cada uma das variáveis mencionadas acima $^{20}$ e a programas de treinamento contra resistência ${ }^{21}$. Obviamente, após encontrar um resultado que não é igual a 5, podemos tentar identificar qual(is) é(são) a(s) causa(s) específica(s) para a perda de ponto, visando definir uma estratégia de treinamento físico para melhorar o resultado. Por exemplo, obesos tendem a ter resultados bem piores ao levantar do que ao sentar (às vezes, diferenças superiores a dois pontos), devido à baixa potência muscular em relação ao peso corporal. Em outra situação, é praticamente impossível que indivíduos com valores de Flexiteste inferiores a 2 nas flexões dorsal e plantar do tornozelo consigam sentar e/ou levantar sem qualquer desequilíbrio e/ou apoio. A utilização do TSL em conjunção com outros procedimentos e medidas pode permitir definição mais precisa dos fatores responsáveis pela perda de pontuação.

\section{Restrições e cuidados}

Ainda que seja um procedimento extremamente simples, recomendamos que o TSL somente seja aplicado por profissionais de saúde (médicos, profissionais de Educação Física, enfermeiros, fisioterapeutas, etc.), não sendo estimulada a autoexecução generalizada. Ressaltamos que a realização do TSL poderá estar contra-indicada em determinados indivíduos idosos, especialmente quando sedentários, ou portadores de alguma condição clínica que demande maior cuidado, tais como pacientes submetidos recentemente a grandes cirurgias, gestantes avançadas, portadores de próteses ou lesões do sistema locomotor em membros inferiores, pacientes com problemas de equilíbrio (ex.: labirintite, etc.) e outros casos semelhantes. Obviamente, maiores cuidados e maior supervisão são necessários para indivíduos de idade avançada ou que possuam pouca ou nenhuma experiência com exercício físico. Em nossa experiência pessoal com mais de 400 avaliações, em indivíduos saudáveis e enfermos entre 3 e 83 anos de idade, não tivemos qualquer acidente ou problema clínico decorrente da aplicação do TSL.

\section{Outros aspectos}

É cada vez maior o interesse sobre a qualidade de vida relacionada com a saúde ${ }^{6,8,9}$. Intervenções terapêuticas, incluindo-se programas de exercício físico, passaram a considerar, mais recentemente, a necessidade de medir e avaliar os efeitos sobre a qualidade de vida ${ }^{9,22,23}$. Nesse sentido, o TSL pode tornar-se um instrumento extremamente interessante e prático para essa finalidade, já que envolve ações que requerem autonomia física mínima. Resultados preliminares de nosso banco de dados mostram que a grande maioria das crianças e adolescentes consegue notas máximas no TSL. A partir dos 25 anos passa a ser progressivamente mais comum a perda de meio a um ponto. Indivíduos com mais de 45 anos, especialmente quando sedentários e com excesso de peso, tendem a perder um a dois pontos, especialmente no ato de levantar, muito embora não seja raro que indivíduos com mais de 60 anos, fisicamente ativos e sem excesso de gordura corporal e ainda bem flexíveis, consigam notas máximas.

\section{CONCLUSÃO}

Ao longo dos últimos anos, temos desenvolvido e proposto vários procedimentos para avaliação em Medicina do Exercício e do Esporte ${ }^{16,24,25}$. O presente artigo apresenta um novo procedimento, correspondendo a uma proposta metodológica para avaliação simplificada e de rápida execução da aptidão muscular funcional mínima para a autonomia. Com a inclusão do TSL no exame físico de consultório ou pré-participação em programa de atividade física, torna-se possível para o profissional de saúde diagnosticar alterações importantes da aptidão muscular funcional e prontamente repassar esta informação ao avaliado. Esta interpretação será ainda mais refinada quando os valores normativos populacionais, ora em coleta, se tornarem universalmente disponíveis.

Considerando a simplicidade na execução e avaliação do procedimento, acredita-se que, com base nos resultados do TSL, possa ser mais fácil para o profissional de saúde sensibilizar os indivíduos avaliados a adotarem estilos de vida mais ativos e orientar de forma mais científica a prática da atividade física regular. Em adendo, aumentam-se as possibilidades de determinação de objetivos específicos, quando da orientação e acompanhamento de programas de exercício físico.

\section{AGRADECIMENTOS}

O autor expressa o seu agradecimento aos Profs. Denise Sardinha Mendes Soares de Araújo e Vitor Agnew Lira pelas sugestões metodológicas e pelas colaborações nas coletas de dados que subsidiaram a proposição definitiva deste teste. Agradecemos ainda os comentários e sugestões do Prof. Dr. Paulo de Tarso Veras Farinatti ao presente artigo. 


\section{REFERÊNCIAS}

1. Blair SN, Kampert JB, Kohl HW 3rd, Barlow CE, Macera CA, Paffenbarger RS Jr, et al. Influences of cardiorespiratory fitness and other precursors on cardiovascular disease and all-cause mortality in men and women. JAMA 1996;276:205-10.

2. Paffenbarger RS Jr, Lee IM. Physical activity and fitness for health and longevity. Res Q Exerc Sport 1996;67(Suppl 3):S11-28.

3. Fletcher GF, Balady G, Froelicher VF, Hartley LH, Haskell WL, Pollock ML. Exercise standards: a statement for healthcare professionals from the American Heart Association. Circulation 1995;91:580-615.

4. Pate RR, Pratt M, Blair SN, Haskell WL, Macera CA, Bouchard C, et al. Physical activity and public health. A recommendation from the Centers for Disease Control and Prevention and the American College of Sports Medicine. JAMA 1995;273:402-7.

5. Pollock ML, Gaesser GA, Butcher JD, Després J-P, Dishman RK, et al. The recommended quantity and quality of exercise for developing and maintaining cardiorespiratory and muscular fitness, and flexibility in healthy adults. Med Sci Sports Exerc 1998;30:975-991.

6. Buchner DM. Physical activity and quality of life in older adults. JAMA 1997;277:64-66.

7. Cramer JA, Spilker B. Quality of life pharmaeconomics - An introduction. Philadelphia: Lippincott-Raven, 1998.

8. Rejeski WJ, Brawley LR, Shumaker SA. Physical activity and healthrelated quality of life. Exerc Sport Sci Rev 1996;24:71-108.

9. Treasure $\mathrm{T}$. The measurement of health related quality of life. Heart 1999;81:331-2.

10. Ferrans C, Powers M. Psychometric assessment of the Quality of Life Index. Res Nurs Health 1992;15:29-38.

11. Stewart A, Hays R, Ware JJ. The MOS short-form general health survey: reliability and validity in a patient population. Med Care 1988; 26:724-35

12. Shephard RJ, Bouchard C. Physical components of fitness: relationship to physical activity and lifestyle. Can J Appl Physiol 1994; 19: 200-14.

13. Fletcher RH, Fletcher SW, Wagner EH. Clinical epidemiology: the essentials. $3^{\text {rd }}$ ed. Philadelphia: Williams \& Wilkins, 1996.

14. Friedland DJ, Go AS, Davoren JB, et al. Evidence-based Medicine - A framework for clinical practice. Stanford: Appleton \& Lange, 1998.

15. Cooper KH. A means of assessing maximal oxygen intake. Correlation between field and treadmill testing. JAMA 1968;203:201-4.
16. Araújo CGS. Flexiteste - Uma nova versão dos mapas de avaliação. Kinesis 1986;2:251-67.

17. Araújo CGS, Araújo DSMS, Lira VA. Teste de sentar-levantar: fidedignidade interavaliadores - Resultados preliminares. In: Anais do XXI Simpósio Internacional de Ciências do Esporte; 8 a 11 de outubro de 1998; São Paulo, Brasil. São Paulo: Celafiscs, 1998, p. 95.

18. Lira VA, Araújo CGS. Teste de sentar-levantar: estudo da variabilidade dia a dia. In: Anais do XXI Simpósio Internacional de Ciências do Esporte; 8 a 11 de Outubro de 1998; São Paulo, Brasil. São Paulo: Celafiscs, 1998, p. 94.

19. Lira VA, Araújo DSMS, Coelho CW, Araújo CGS. Sitting-rising test Inter-observer reliability results. In: Proceedings of the $46^{\text {th }}$ Annual Meeting of The American College of Sports Medicine; 1999 June 2-5; Seattle, USA. Med Sci Sports Exerc 1999;31(Suppl 5):S78.

20. Silva EB, Lira VA, Araújo CGS. Teste de sentar-levantar: efeito de variações abruptas do peso corporal em indivíduos jovens ativos. In: Anais do XXI Simpósio Internacional de Ciências do Esporte; 8 a 11 de Outubro de 1998; São Paulo, Brasil. São Paulo: Celafiscs, 1998, p. 93.

21. Coelho CW, Velloso CR, Brasil RRLO, Conceição FL, Vaisman M, Araújo CGS. Six-week home-based resistance training improves muscle power in adult patients with GH-deficiency. In: Proceedings of the $46^{\text {th }}$ Annual Meeting of The American College of Sports Medicine; 1999 June 2-5; Seattle, USA. Med Sci Sports Exerc 1999;31(Suppl 5): S268.

22. D'Ambrosio CD, Bowman T, Mohsenin V. Quality of life in patients with obstructive sleep apnea - Effect of nasal continuous positive airway pressure: a prospective study. Chest 1999;115:123-9.

23. Engebrestson TO, Clark MM, Niaura RS, Phillips T, Albrecht A, Tilkemeier P. Quality of life and anxiety in a phase II cardiac rehabilitation program. Med Sci Sports Exerc 1999;31:216-23.

24. Araújo CGS, Nóbrega ACL, Castro CLB. Heart rate responses to deep breathing and 4-seconds of exercise before and after pharmacological blockade with atropine and propranolol. Clin Auton Res 1992;2: 35-40.

25. Araújo CGS, Pável RC, Gomes PSC. Comparison of somatotype and speed in competitive swimming at different phases of training. In: Terauds J, Bedingfield EW, editors. Swimming III. Baltimore: University Park Press, 1979:329-37. 